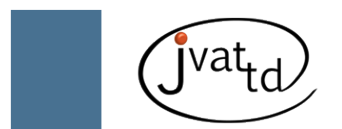

\title{
About a case of blindness following scorpion envenomation
}

\author{
Delma K (1)
}

(1) Service of Anesthesia and Reanimation, Ouargla Hospital, Ouargla, Algeria.

\begin{abstract}
The author reports a case of blindness occurred after three scorpion stings in a young woman from the region Ouargla, Algeria. The absence of signs of neurological and cardiovascular envenomation and the functional examinations of eyes is likely to be the consequence of a toxic neuropathy. Two months later, blindness persists and functional prognosis remains reserved.
\end{abstract}

Key words: scorpion sting, blindness, toxic neuropathy.

\section{INTRODUCTION}

In the region of Ouargla, south-east of Algeria, scorpion stings are frequent and often severe, especially in children, despite a significant improvement in scorpion stings treatment in recent years (1-3).

If case fatality rate remains relatively low, the majority of deaths occur following a cardiocirculatory failure, including acute pulmonary edema. It is also described some rare cerebral vascular accidents after scorpion sting $(4,5)$. These may be the result of a venom direct action or consequence of hemodynamic and blood coagulation disorders.

This observation shows an exceptional complication, namely blindness, occurred after scorpion sting.

\section{CASE REPORT}

A young woman, aged 24, stung by a scorpion, consulted the emergency ward of Ouargla hospital on June 24th, 2012 soon after the sting. She showed no neurological or cardiovascular symptoms. The envenomation was graded 2 , that is to say, local pain at the sting site and digestive disorders (vomiting, asthenia). Blood pressure was $90 / 50 \mathrm{mmHg}$ and the patient had hypoglycemia $\left(0.40 \mathrm{~g} \cdot \mathrm{L}^{-1}\right)$ treated with infusion of hypertonic glucose solution. According to therapeutic protocol in force in Algeria, she received the scorpion antiserum (Institut Pasteur, 1 vial intramuscularly) with corticosteroids, and paracetamol (intravenously, $1 \mathrm{~g}$ every 6 hours until pain disappearance). She was rapidly discharged from the hospital.

The next day she returned due to another 
scorpion sting, without systemic clinical signs. After the same treatment as the day before and a short clinical observation time, she went home again.

Two days later, she came again to the emergency room because of a third scorpion sting, showing similar symptoms to the day before. She was treated according to the same protocol as the two previous occasions. The progression was favorable and quickly the young woman discharged from the hospital.

Twenty-four hours after the third discharge, she wakes from a nap completely blind. She returned to the hospital and was hospitalized in the ophthalmological service.

She reports no medical history, including regarding ophthalmology. She did not consult traditional therapist, and she said not taken any treatment other than those which had been administered in the hospital.

At admission, she showed a good general condition, an estimated Glasgow Coma Scale at $15 / 15$ without neurological disorder, a stable hemodynamic condition, and a normal cardiorespiratory function, with no blood coagulation disorders. Renal function was normal.

The ophthalmological evaluation showed visual acuity less than $1 / 10^{\text {th }}$ with questionable light perception and oculomotor palsy. Fundus examination and cerebral tomodensitometry were normal.

High-dose corticosteroids (dexamethasone intravenously, $80 \mathrm{mg}$ every 6 hours, during 5 days, then 80 mg every 8 hours during 8 days and, finally, Cortancyl 5 mg per oral route during 6 weeks), combined with citicoline (200 mg every 12 hours per oral route, during 2 days) were administered without any results. The patient was transferred to the Department of Ophthalmology of the University Medical Center of Beni Messous, in Algiers, for further investigations and appropriate care.

Electroencephalography and orbitofrontal brain magnetic resonance imaging were performed (Figures 1 and 2). Both were normal.

Visual evoked potential (VEP) showed bilateral involvement of VEP, large and small checkered, with preservation of normal latency reflecting an axonal damage of the optic nerves, consistent with a toxic neuropathy (Figures 3 and 4).

Flash VEP were present, predominant on the

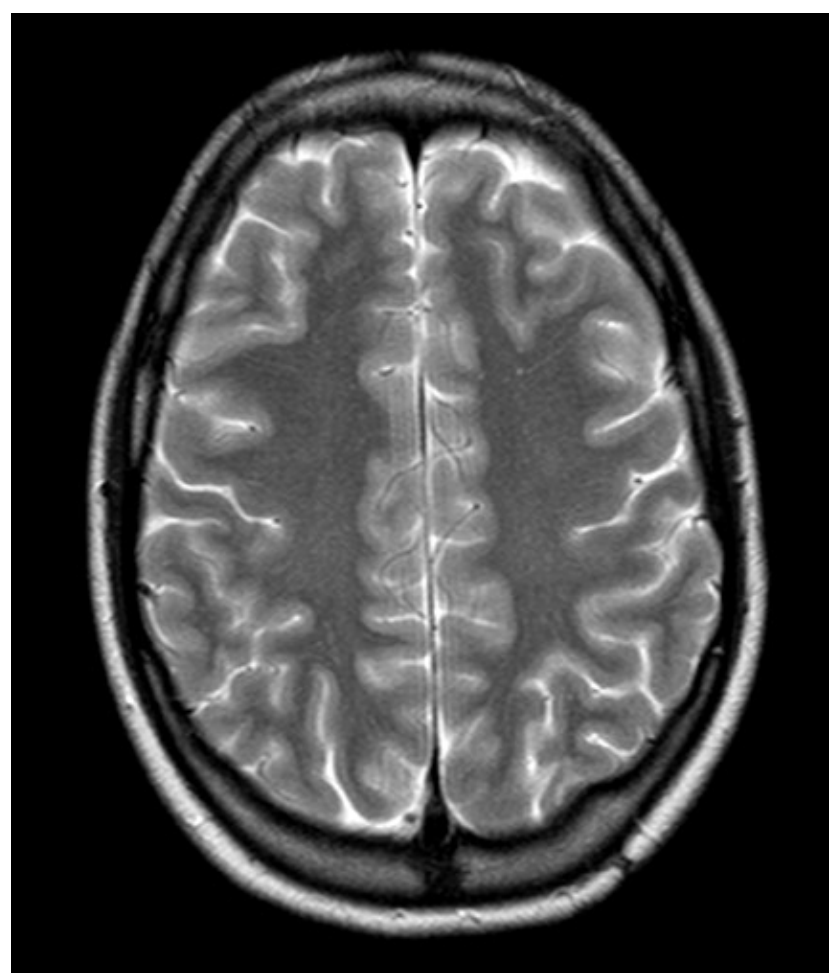

Figure 1. Brain magnetic resonance imaging: transverse section.

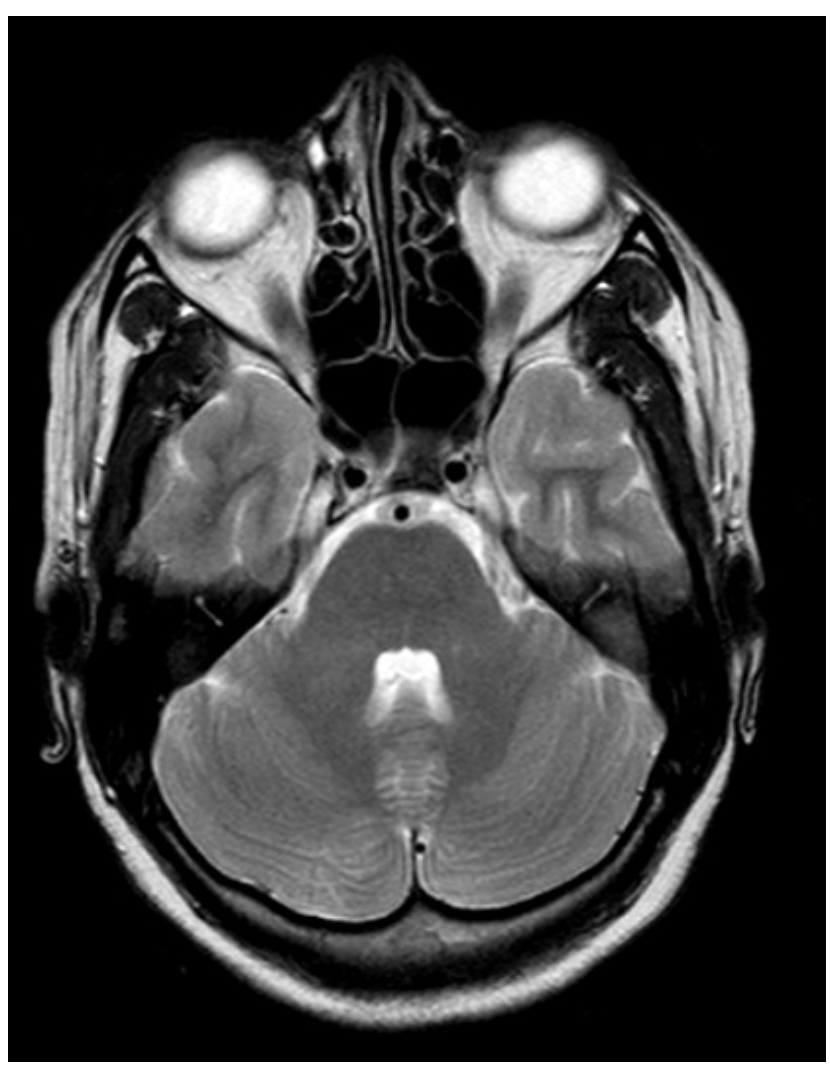

Figure 2. Brain magnetic resonance imaging: orbitofrontal section. 


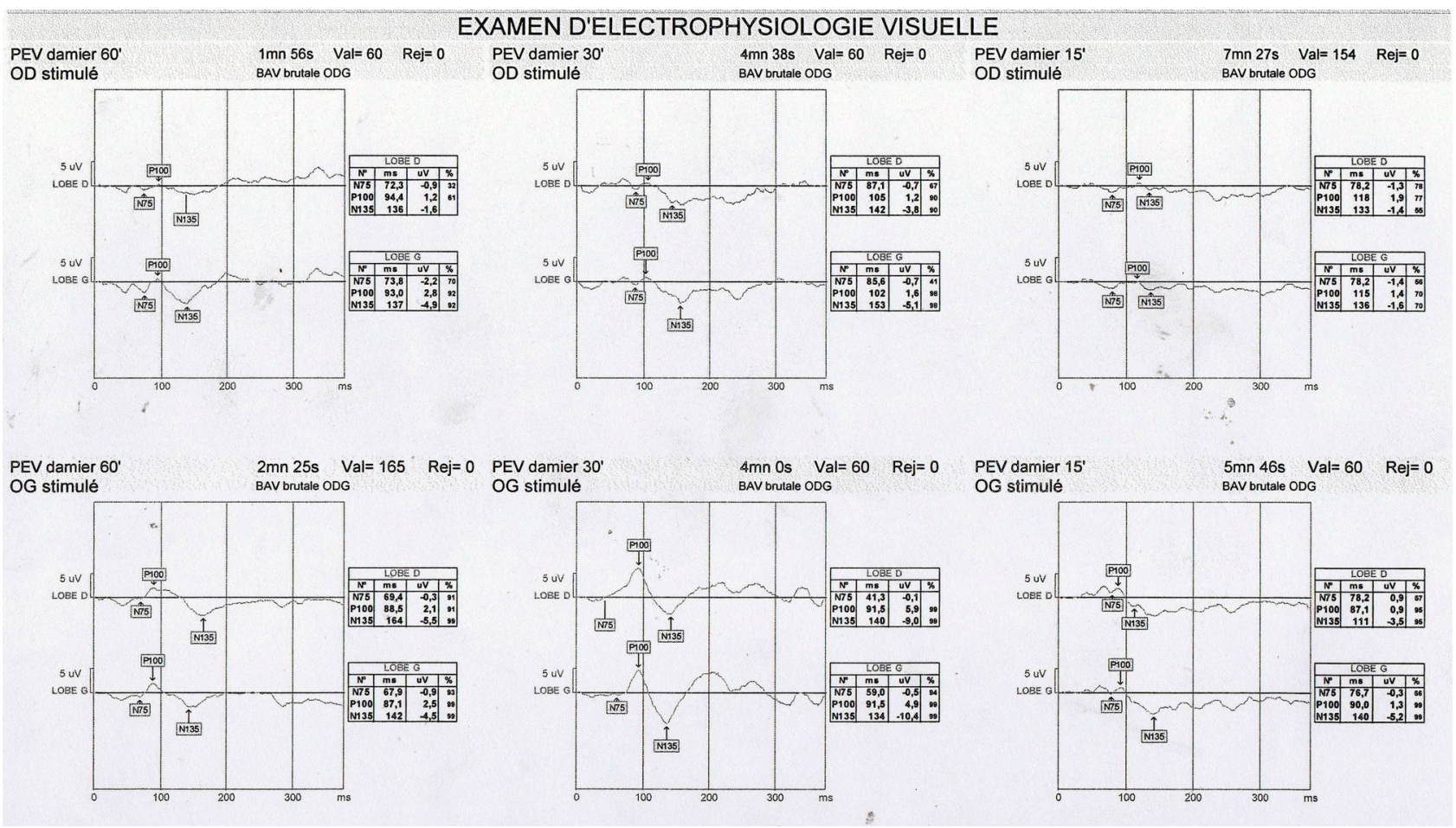

Figure 3. Functional visual exploration: checkerboard visual evoked potential.

right side, showing no severe impairments of visual pathways (Figure 5); however, this did not exclude the possibility of cortical blindness.

The diagnosis of toxic neuropathy was retained. The patient was treated with corticosteroids. There is no improvement to date.

On October 31, 2012, ophthalmological examination showed a poor visual acuity in both eyes (counting fingers at 1 meter), but a normal eye movements. Eye fundus (both macula and papilla) was normal.

\section{DISCUSSION}

This case of blindness following a scorpion sting differs from the other two we found in the literature, mainly by the lack of other neurologic or cardiovascular symptoms. Roussel (6) mentioned an African case which presented a few weeks transient blindness, after a severe neurological syndrome involving fever, loss of consciousness, convulsions and secondary pyramidal syndrome. Sengupta et al. (7) described a scorpion envenomation in India with acute pulmonary edema, acute renal failure, and coma. They conclude that blindness occurred as a consequence of diffuse infarctions of both cerebella and brain.

Here, the young lady showed an isolated blindness, without any other neurologic or cardiovascular symptoms able to explain this complication. Several hypotheses have been proposed by ophthalmologists to explain this toxic neuropathy:

- the direct action of scorpion venom on the optic nerve cannot be excluded, although this has never been reported to our knowledge;

- an adverse event related to the treatment administered to the patient (scorpion antivenin, paracetamol or corticosteroids) is possible, although no other signs of intolerance have been observed;

- finally, considering the number - quite exceptional - of scorpion stings three consecutive days, it is also possible to talk about a phenomenon of hypersensitivity to scorpion venom inoculation, despite a minimal quantity of inoculated venom referring to the absence of systemic symptoms. 


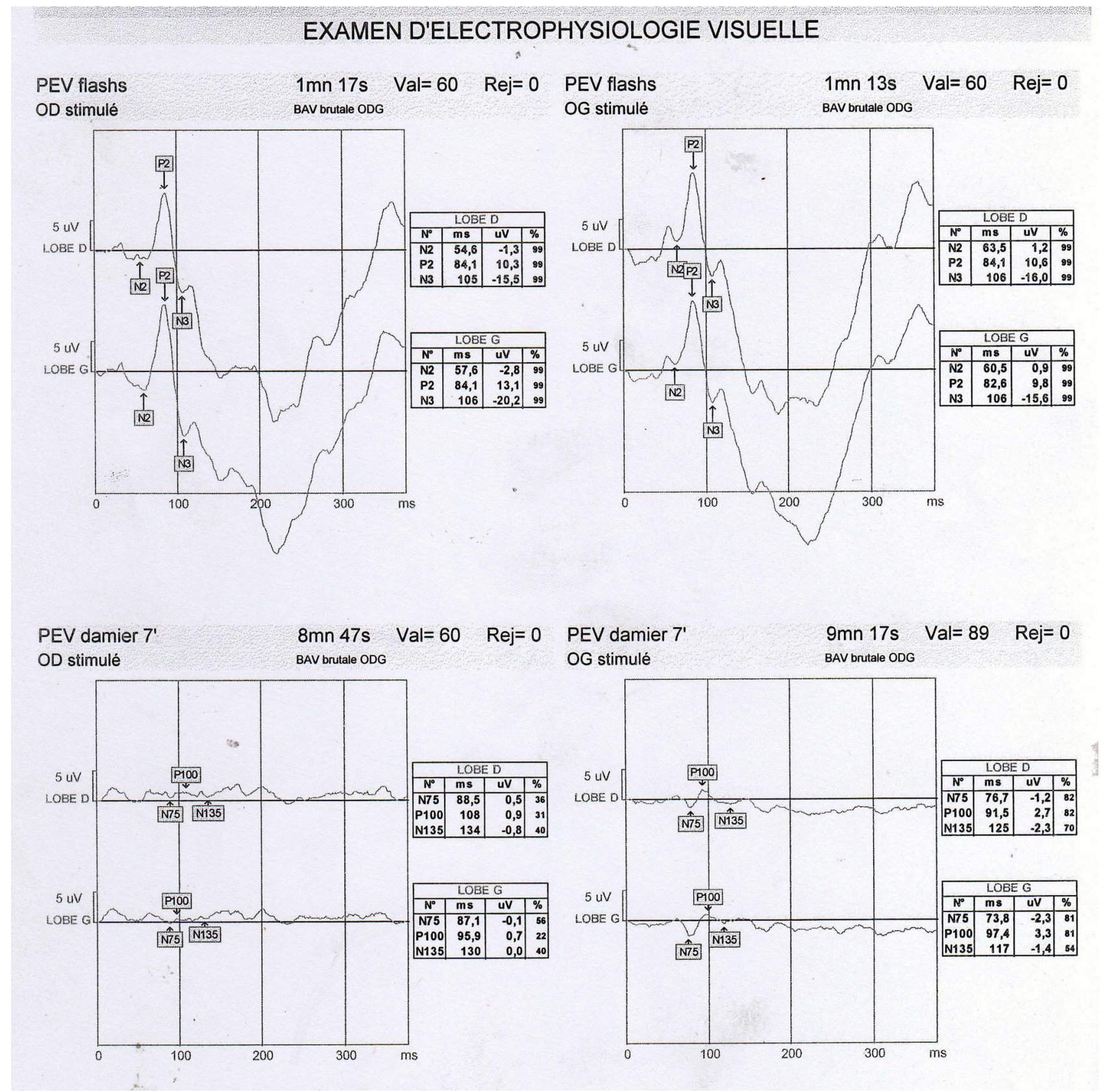

Figure 4. Functional visual exploration: flash visual evoked potential. 


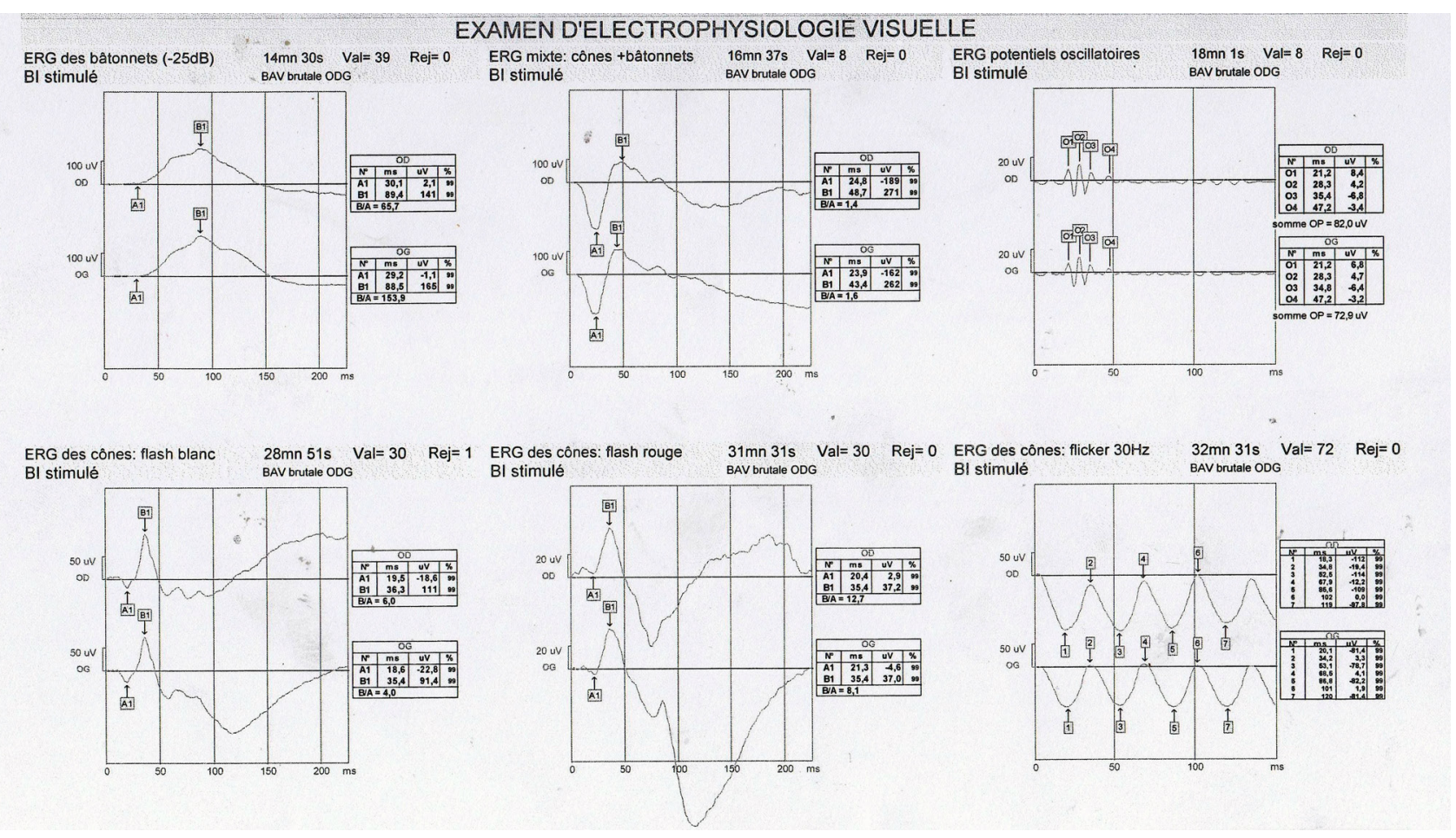

Figure 5. Functional visual exploration: flash visual evoked potential.

\section{COPYRIGHT}

(c) CEVAP 2012

\section{SUBMISSION STATUS}

Received: September 28, 2012.

Accepted: November 8, 2012.

Abstract published online: November 8, 2012.

Full paper published online: November 30, 2012.

\section{CONFLICTS OF INTEREST}

The authors declare no conflicts of interest.

\section{FINANCIAL SOURCE}

The Ouargla Hospital, Algeria, supported the present study.

\section{CORRESPONDENCE TO}

Delma Kilani, Service d'Anesthésie Réanimation, Hôpital d'Ouargla, Algérie, BP 89 Gharbouz Ouargla 30032 Algeria. Phone: 213771313942. Fax 213 29762071. Email: kilani.delma@gmail. com.

\section{REFERENCES}

1. Benguedda AC, Laraba-Djébari F, Ouahdi M, Hellal H, Griene L, Guerenik M, Laid Y. Expériences de quinze années de lutte contre l'envenimation scorpionique en Algérie. Bull Soc Pathol Exot. 2002;95(3);205-8.

2. Hellal H, Guerinik M, Griene L, Laid Y, Mesbah S, Merad R, Alamir B.Données épidémiologiques sur l'envenimation scorpionique en Algérie. Bull Soc Pathol Exot. 2012;105(3):189-93.

3. Chippaux JP, Goyffon M. Epidemiology of scorpionism: a global appraisal. Acta Tropica. 2008;107(1):71-79.

4. Udayakumar N, Rajendiran C, Srinivasan AV. Cerebrovascular manifestations in scorpion sting: a case series. Indian J Med Sci. 2006;60(6):241-4.

5. Derkaoui A, Elbouazzaoui A, Lfraiji Z, Achour S, Labib S, Harandou M. Accident vasculaire cérébral ischémique: une complication rare de l'envenimation scorpionique. Presse Med. 2011;40(1 Pt 1):106-8.

6. Roussel L. Scorpionisme compliqué par une cécité transitoire. A propos d'un cas. Med Trop. 1986;46(4):409-11.

7. Sengupta S, Dhanapal P, Ravindran RD, Devi N. Cerebral blindness after scorpion sting. J Neuroophthalmol. 2009;29(2):154-5. 